http://jmscr.igmpublication.org/home/ ISSN (e)-2347-176x ISSN (p) 2455-0450 crossref DOI: https://dx.doi.org/10.18535/jmscr/v9i4.27

\title{
Prevalent non-tuberculous Mycobacteria (NTM) species among presumptive MTBDR patients from peripheral health facilities referred for testing at KEMRI TB Laboratory-Kisumu, Kenya
}

\author{
Okumu Albert ${ }^{1}$, Asito Stephen ${ }^{2}$, Omondi Isaiah ${ }^{1}$, Ogollah Christine ${ }^{1}$, Shiluli Clement ${ }^{4}$, \\ Murithi Wilfred $^{1}$, Odero Ronald ${ }^{1}$, Malika Timothy ${ }^{3}$, Wandiga Steve ${ }^{1}$ \\ ${ }^{1}$ Kenya Medical Research Institute (KEMRI) Center for Global Health Research, Kisumu, Kenya \\ ${ }^{2}$ Jaramogi Oginga Odinga University of Science and Technology, JOOUST, Bondo, Kenya \\ ${ }^{3}$ Jaramogi Oginga Odinga Teaching and Referral Hospital, JOOTRH, Ministry of Health, Kisumu-Kenya \\ ${ }^{4}$ UZIMA University, Microbiology Department, Kisumu, Kenya
}

\begin{abstract}
Objectives: The aim of this study was to establish prevalent Nontuberculosis Mycobacteria among presumptive multidrug resistant TB (MDRTB) patients in western Kenya. Sputum samples obtained from laboratory confirmed MDRTB patients were processed using standard culture methods, fluorescence microscopy (FM), MGIT culture, Xpert MTB/RIF. Mycobacteria tuberculosis complex (MTBc) and NTM were identified using line probe assays techniques.

Results: Between January 2012 to December 2015, a total of 287 samples from presumptive MDRTB individuals were flagged positive with liquid culture (MGIT), subsequently had line probe assay (LPA) performed for potential nontuberculous mycobacteria species. Of these, 155 (54\%) had NTMs and 14 (4.87\%) MTBc by LPA technique, respectively. Males constituted 140(49\%) of which 64 (22.3\%) were HIV infected, while females were a total of 160(55.7\%), with 96 (33.4\%) HIV infected. 41(14.3\%) individuals with unknown HIV status. M. intracellulare 62(21.6\%), and M. scrofulaceum 13(4.5\%) were most prevalent NTMs species identified.

Keywords: Nontuberculous mycobacteria, multidrug resistance tuberculosis, pulmonary, diagnosis, line probe assay, microscopy.
\end{abstract}

\section{Introduction}

In 2019, there were an estimated 10 million new (incident) Tuberculosis (TB) cases worldwide, of which 5.6 million (56\%) were among men, 3.2 million (32\%) among women and 1.2 million $(12 \%)$ among children ${ }^{(1)}$. Globally, an estimated 1.4 million TB deaths were reported in 2019; 0.2 million of these deaths occurred in people living with HIV $(\mathrm{PLWH})^{(1)}$. In addition, $8.2 \%$ of new TB cases reported globally occurred in PLWH in $2019^{(1)}$. Simultaneous infection with HIV and Mycobacterium tuberculosis (MTB) is a deadly combination affecting large populations in
Africa, Asia, Latin America and Eastern Europe. Progressive immune dysfunction caused by HIV infection increases susceptibility to MTB infection as well as progression from latent infection to active tuberculosis (TB) disease ${ }^{(2)}$.

More recently, other species of mycobacterium causing clinical disease have been identified in many geographical regions where they cause significant disease burden than $\mathrm{MTB}^{(3)}$. Mycobacteria other than tuberculous (MOTT), Mycobacteria species and $M$. leprae are known as nontuberculous mycobacteria (NTM), previously, they were referred to as "atypical" mycobacterial 
species and they share many common properties with MTB, such as acid fastness, ability to cause pulmonary and extra-pulmonary granulomatous disorders and pathogenicity ${ }^{(4)}$. These properties vary among different species and may cause opportunistic infections, however, the lung seems to be their most common site of involvement, same as $\mathrm{MTB}^{(5,6)}$.

Although MTB and NTM cause chronic lung infections (7), only MTB spreads from person to person by inhalation of organisms expectorated into the air. NTM infections are acquired directly from environment, where they are often present in soil and various water sources, thus considered opportunistic pathogens ${ }^{(8)}$. Since pathologic findings of NTM and MTB regarding granulomatous inflammation and even cavity are very similar, it is difficult to distinguish them from pulmonary $\mathrm{TB}^{(9)}$.

Mycobacterium tuberculosis diagnosis is an important disease control tool, consisting of both conventional methods (acid-fast microscopy, a primary procedure in most health facilities, culture, biochemical identification, antituberculosis drug-susceptibility testing (AST) and modern molecular techniques, hence laboratory plays an important role in diagnosing tuberculosis (TB) and the identification and drug sensitivity testing (DST) of Mycobacterium tuberculosis ${ }^{(10)}$.

Kenya is considered a high TB-burden country, with a TB prevalence of 553/100,000 (KTBPS, 2016) and with limited access to mycobacterial culture. $95 \%$ of pulmonary bacterial infections are due to Mtb while $5 \%$ by NTM (11). Western region has a TB prevalence of $14 \%$, twice the national rates making TB/HIV co-infection a serious health concern ${ }^{(12)}$.

The incidence of disease caused by NTM is somewhat independent of that of TB, but is determined by the number, distribution, and species of NTM in the environment ${ }^{(13)}$ and susceptibility of human population. Little is known about the relative burden of $\mathrm{NTMs}^{(13)}$, including Kenya, where public laboratories performing bacteriological cultures, primarily test for MTBc but seldom test for NTMs.

The reports of nontuberculous mycobacteria (NTM) associated with extrapulmonary diseases are increasing in tertiary care hospitals. Despite a significant increase in knowledge about NTM infections, they still represent a diagnostic and therapeutic challenge ${ }^{(14)}$.

The high costs to the patient and society should lead health care providers to consider NTM in all patients suspected of having $\mathrm{TB}^{(15)}$. Even in low resource countries where mycobacterial culture and molecular identification procedures are not routinely practiced, there could be great saving of country resources associated with correctly diagnosing and treating patients. It is another reason for National TB Programs to integrate mycobacterial culture and molecular identification procedures into routine practice ${ }^{(15)}$.

Identifying NTM frequency and alerting physicians to this problem may increase awareness, help better understand the epidemiology, get patients on appropriate therapy sooner, and decrease the stigma and cost of this care $^{(16)}$.

In our demonstration of NTMs, we used line probe assay kits from HAIN LIFESCIENCE, which provides for both the common mycobacteria (CM) and atypical species (AS) of mycobacteria species. These kits have the ability to distinguish the various types of species of mycobacteria of interests ${ }^{(17)}$. The molecular based technologies that provide the genomic identification and demonstrates the presence of the corresponding DNA for mycobacteria species ${ }^{(18)}$. In 2012, the Kenya's MOH TB program scaled up MDRTB surveillance across the country, by authorizing KEMRI CGHR TB laboratory to support with culture and resistance testing for presumptive multidrug resistant patients in Western and north rift region of Kenya. The study aimed to show the prevalent NTMs in these populations through the various techniques employed. 


\section{Main Text}

NTM presence shows positivity with common routine Ziehl Nelsen (ZN) microscopy methods available hence patients may routinely be initiated on wrong medications due to MTBc. Hence parallel diagnosis for tuberculosis diagnosis is required to classify $\mathrm{MTBc}$ to inform patient management.

\section{Methods}

\section{The Study Design}

This was a cross sectional study, where samples were collected at one-time point and sent for culture. Stored sputum samples collected from presumptive MDRTB patients western Kenya, were processed for and determined for MTBc and NTM presence.

These are patients who had been initiated into the TB programs, primary regimen but did not show signs of improvement.

\section{Laboratory Procedures}

Sputum samples were collected from January 2012 through December 2015 as part of Multidrug resistance surveillance, an activity by the $\mathrm{MoH}$ through the peripheral facilities. Patients were asked to produce at least one sputum sample during their hospital visits. These samples were then packaged and transported via courier delivery or hand delivered to the TB laboratory for processing.

In the laboratory, samples were processed based on the national TB program testing algorithm. All presumptive MDRTB cases, had Xpert Mtb tests, smear microscopy (ZN/FM), and all positive smears had Line probe assays (LPA) tests as well as MGIT cultures of which positives had MTBc identification carried thereafter drug susceptibility tests for first line drugs.

During processing, samples were digested and decontaminated using $\mathrm{N}$-acetyl-L-cysteine/ $4 \%$ sodium hydroxide-sodium citrate (NALC/NaOH Na-citrate, [final concentration of $\mathrm{NaOH} 1 \%$ ]), neutralized with $\mathrm{pH} 6.8$ phosphate buffer solution (PBS), concentrated through centrifugation @ $3,000 \mathrm{x} \mathrm{g}$ for 15 minutes, decanted, and resuspended in $2.0 \mathrm{~mL}$ of fresh PBS. Thereafter inoculated into MGIT tubes, a drop (0.03ul) for smear preparations, $0.5 \mathrm{ul}$ aliquoted for Xpert Mtb Rif PCR and also LPA (HAIN CM\& AS) kits. The tubes were incubated at $37^{\circ} \mathrm{C}$ per culture SOP, in the BACTEC 960 and observed for any growths per, subsequent positive flags were processed for identification using Brain heart infusion (BHI) and Ziehl Nelsen smear.

Microscopic evaluations were performed on all tubes showing positive flags, as well as on BHI culture plates for confirmation of AFB; growth consistent with mycobacteria was identified using an immunochromatographic assay (MGIT TBc ID, BD Sparksville Maryland, MD). All positive smears, were processed using Line probe assays, the Genotype Mycobacterium Common mycobacteria or Atypical species (CM/AS) line probe assay (Hain LifeScience, Nehren, Germany). Quality control procedures included inoculation of MGIT with processed artificial sputum (AS) spiked with an American Type Culture Collection (ATCC) - H37rv control strain for positive control and non-spiked AS for negative control.

\section{Classification of Culture Results}

The MTBC yield was compared to the prevalent NTMs identified through the performance of Line probe assay (LPA) with respect to contamination, NTMS and MTBC yield. The final outcome was categorized as: MTBC positive, negative, contaminated, or NTM positive. Cultures were considered MTBC positive if growth obtained from the cultures was identified as such using the immunochromatographic assay, negative if no growth was observed after the standard 6 weeks (42 days) of incubation, contaminated if only nonacid-fast organisms grew, and NTM if growth was negative for MTBC by the immunochromatographic assay and identified as NTM using the line probe assay. The NTMs were identified and classified into the various species using HAIN (CM\&AS) kits.

\section{Statistical Analysis}

Participant data retrieved from source documents were keyed in and stored in a password-protected 
Laboratory Information System (LIMS). Data was analysed using STATA version 14 for Windows. Frequencies and percentages were used to present the participant characteristics, HIV results and the MTB results for HAIN CM/AS, Ziehl Neelsen $(\mathrm{ZN}) /$ Fluorescent (FM) microscopy and Xpert Mtb rif PCR.

\section{Ethical Review}

We sought ethical approval from KEMRI's IRB (SERU) to conduct this study. As part of collaboration with $\mathrm{MOH}$, we also had a MoU with National TB Leprosy and lung diseases program, which permitted use of left over samples, these presented No or minimal risk to participants. Patients' confidentiality was adhered to maintained and protected, during the study.

\section{Results}

Table 1. A total of 287 samples flagged positive on liquid culture (MGIT), WHO gold standard procedures, were processed for presumptive MDRTB patients using a molecular technique (line probe assay), to determine potential nontuberculous mycobacteria species. The demographic characteristics of these participants with NTMs, 147(51.2\%) were females and $140(48.8 \%)$ males. Mean age was 38.1 (14.8\%), and a prevalence of MTBc of $62(21.6 \%)$. HIV positive individuals with NTMs were 156 (54.4\%), 92(32.1\%) and 39 (13.6\%) with no HIV status indicated.

Table 2 shows risk factors associated with NTM infection, where gender showed a significance of p-v 0.036, with an OR (0.77) while HIV status was insignificant, with an OR (0.89) and a p-v $0.225 .167(58 \%)$ individuals had comorbidity of NTM to either Mtb and/or HIV or both. $26(0.6 \%)$ had NTM only, 84 (2.1\%) had both NTM and Mtb co-infections.

Table 4 shows the prevalent mycobacteria species in relation to HIV status and gender. Of the species identified, Mycobacteria intracellulare were $62(22.1 \%)$, of which $37(66 \%)$ among HIV positive, and 33(53.3\%) in males. Mycobacteria scrofulaceum were $13(4.5 \%)$, of $9(69 \%)$ from
HIV infected, 7(30\%) and 6 (30\%) among females and males respectively. Of note, other high grade cocci (HGC) organisms were prevalent, 45(16\%) were identified of which 29(70.7\%) were HIV infected, 25(55.6\%) and 20(44.4\%) were females and males respectively. Other NTMs identified were Mycobacterium fortuitum 11(3.8\%), Mycobacteria celatum 10(3.5\%), Mycobacteria abscessus 7(2.5\%), Mycobacterium gordonae 7(2.5\%), Mycobacterium malmoense 6(2,14\%), Mycobacterium avium 3(1.1\%), and Mycobacterium interjectum and Mycobacterium kansasii each having $1(0.3 \%)$ respectively.

Table 5 shows the participants having both NTM and Mtb based on their HIV status. Of the 122 $(42.5 \% \%)$ with NTM only, 84(45.6) of them were HIV positive while 26(14.1\%) HIV non-infected. From among $62(21.6 \%)$ individuals with both Mtb and NTM comorbidity, 32(17.3) HIV infected and 25(13.5\%) HIV non-infected.

Table 6 shows the prevalence of NTM species in relation to HIV status and gender. Out of the 287 individuals presumed to have NTMs, 155(55.6\%) had various types of NTMs, with the most prevalent NTMs were 62(21.6\%) M. intracelluare, 23(8\%) Mycobacteria species, 13(4.5\%) $M$. scrofulaceum, 14(4.9\%) MTBC, 10(3.9\%) M. celatum, 7(2.4\%) M. abscessus, 11(3.8\%) M. fortuitum, among others as shown in Table.

Of these individuals, $160(55.7 \%)$ had HIV, of which $15(23.4 \%)$ were males with prevalent NTMs species of $M$. intracellulare, $5(7.8 \%) M$. fortuitum, 4(6.25\%) M. scrofulaceum, 3(4.7\%), among others, and 2(3.1\%) had MTBC while among females, 22(22.9\%) had $M$. intracellulare, 3(3.13\%) M. avium spp, 5(5.2\%) M. scrofulaceum among others. 88(30.7\%)HIV non-infected, in males, $12(22.6 \%)$ had $M$. intracellulare, $11(20.8 \%)$, among others, in females had $6(17.1 \%) M$.intracellulare, among others while $39(13.5 \%)$ with unknown HIV status respectively and MTBC respectively, among others.. 


\section{Appendix: Tables}

Table 1: Participant Characteristics (demographics and HIV status)

\begin{tabular}{|c|c|c|}
\hline \multirow[b]{2}{*}{ Demographic and risk factors } & \multicolumn{2}{|c|}{ Overall $(\mathrm{n}=287)$} \\
\hline & $\mathrm{N}$ & $\%$ \\
\hline \multicolumn{3}{|l|}{ Gender } \\
\hline Female & 147 & 51.2 \\
\hline Male & 140 & 48.8 \\
\hline Age: Mean (SD) & \multicolumn{2}{|c|}{$38.1(14.8)$} \\
\hline \multicolumn{3}{|l|}{ Age groups (years) } \\
\hline$<20$ & 27 & 9.4 \\
\hline $20-40$ & 128 & 44.6 \\
\hline $40-60$ & 99 & 34.5 \\
\hline$>60$ & 22 & 7.7 \\
\hline Prevalence of MTBC & 62 & 21.6 \\
\hline \multicolumn{3}{|l|}{ Concurrent conditions } \\
\hline HIV +ve & 156 & 54.4 \\
\hline HIV -ve & 92 & 32.1 \\
\hline Unknown & 39 & 13.6 \\
\hline
\end{tabular}

Table 2 Risk factors associated with NTM infection

\begin{tabular}{lccc}
\hline & OR & $95 \%$ CI & p-value \\
\hline Gender & 0.77 & $0.61-0.98$ & 0.036 \\
HIV positive & 0.89 & $0.73-1.08$ & 0.225 \\
\hline
\end{tabular}

Table 3: Comorbidity of NTM with either $M . t b$ and or HIV among individuals with NTMs

\begin{tabular}{lll}
\hline Type of infection & N & $\%$ \\
\hline NTM alone & 26 & 0.6 \\
HIV +NTM & 84 & 2.1 \\
NTM + M.tb & 25 & 0.6 \\
HIV + NTM +M.tb & 32 & 0.8 \\
Total & 167 & \\
\hline
\end{tabular}

Table 4: Comorbidity of NTM with either M.tb and or HIV among individuals with NTMs by Gender

\begin{tabular}{llll} 
& & \multicolumn{2}{c}{ Gender n $(\%)$} \\
\cline { 3 - 4 } Type of infection & $\mathrm{n}$ & Male & Female \\
\hline NTM alone & 26 & $15(0.68)$ & $11(0.60)$ \\
HIV +NTM & 84 & $38(1.72)$ & $46(2.52)$ \\
NTM + M.tb & 25 & $19(0.86)$ & $6(0.33)$ \\
HIV + NTM +M.tb & 32 & $13(0.59)$ & $19(1.04)$ \\
\hline
\end{tabular}

Table 5: Comorbidity of NTM with M.tb by HIV

\begin{tabular}{lcccc} 
Prevalence of NTM and NTM infection & $\mathbf{n}(\boldsymbol{\%})$ & Positive & Negative & Others \\
\hline NTM Only & $122(42.5)$ & $84(45.6)$ & $26(14.1)$ & $12(6.5)$ \\
M.tb + NTM & $62(21.6)$ & $32(17.3)$ & $25(13.5)$ & $5(2.7)$ \\
\hline
\end{tabular}


Table 6: Species distribution of the NTM isolated from PDCs/hospitals by HIV Status and Gender

\begin{tabular}{|c|c|c|c|c|c|c|c|}
\hline \multirow[b]{2}{*}{$\begin{array}{l}\text { Prevalence } \\
\text { infection }\end{array}$} & \multirow[b]{2}{*}{ NTM } & \multirow[b]{2}{*}{$\mathbf{n}$} & \multicolumn{3}{|c|}{ HIV Status n (\%) } & \multicolumn{2}{|c|}{ Gender n(\%) } \\
\hline & & & Positive & Negative & Others & Male & Female \\
\hline M. gordonae & & 5 & $4(2.50)$ & $1(1.14)$ & 0 & $4(2.86)$ & $1(0.68)$ \\
\hline M. Abscessus & & 7 & $1(0.63)$ & $3(3.41)$ & $3(7.69)$ & $1(0.71)$ & $6(4.08)$ \\
\hline M Asiasticum & & 4 & $1(0.63)$ & $2(2.71)$ & $1(2.56)$ & $1(0.71)$ & $3(2.04)$ \\
\hline M. Genavense & & 2 & $1(0.63)$ & $1(1.14)$ & $5(12.82)$ & $1(0.71)$ & $1(0.68)$ \\
\hline M. interjectum & & 1 & 0 & $1(1.14)$ & 0 & 0 & $1(0.68)$ \\
\hline M. intracellulae & & 62 & $37(23.1)$ & $18(20.45)$ & $7(17.95)$ & $31(22.14)$ & $31(21.09)$ \\
\hline M. scrofulaceum & & 13 & $9(5.63)$ & $4(4.55)$ & 0 & $6(4.29)$ & $7(4.76)$ \\
\hline M. Mucogenum & & 2 & 0 & $2(2.27)$ & 0 & $2(1.43)$ & 0 \\
\hline M. Smegmatis & & 2 & $1(0.63)$ & 0 & $1(2.56)$ & 0 & $2(1.36)$ \\
\hline MTBC & & 14 & $7(4.38)$ & $3(3.41)$ & $4(10.26)$ & $7(5.00)$ & $7(4.76)$ \\
\hline Myco SPP & & 23 & $15(9.38)$ & $6(6.82)$ & $2(5.13)$ & $12(8.57)$ & $11(7.48)$ \\
\hline M. avium spp & & 3 & $3(1.88)$ & 0 & 0 & 0 & $2(3.04)$ \\
\hline M. fortuitum & & 11 & $6(3.75)$ & $3(3.41)$ & $2(5.13)$ & $8(5.71)$ & $3(2.04)$ \\
\hline M. kansasii & & 1 & 0 & $1(1.14)$ & 0 & $1(0.71)$ & 0 \\
\hline M. lentiflavum & & 2 & $1(0.63)$ & $1(1.14)$ & 0 & 0 & $2(1.36)$ \\
\hline High GC & & 49 & $28(17.5)$ & $15(17.05)$ & $6(15.38)$ & $24(17.14)$ & $25(17.01)$ \\
\hline M. celatum & & 10 & $4(2.50)$ & $1(1.14)$ & $5(12.82)$ & $5(3.57)$ & $5(3.40)$ \\
\hline M. malmoense & & 5 & $4(2.50)$ & 0 & $1(2.56)$ & $4(2.86)$ & $1(0.68)$ \\
\hline NTM not identified & & 58 & $30(18.75)$ & $23(26.14)$ & $5(12.82)$ & $29(20.71)$ & $29(19.73)$ \\
\hline Negative & & 13 & $8(5.00)$ & $3(3.41)$ & $2(5.13)$ & $4(2.86)$ & $9(6.12)$ \\
\hline
\end{tabular}

\section{Discussions}

NTM have been increasingly recognized as an important cause of morbidity in the developing countries $^{(14)}$. The identification of NTM is important because positive microscopy cannot differentiate $M$. tuberculosis complex from NTM infection, causing diagnostic and clinical dilemmas. Management of patients with MTBC and NTM is entirely different; therefore, prompt isolation, detection, and differentiation are necessary for suitable management ${ }^{(6,14,19)}$

In this study, the prevalence of NTMs among presumptive MDR patients was $3.7 \%$ lower than a study done in $\operatorname{Iran}^{(15)}$ which found $30 \%$ of NTMs among MDR TB patients. Similarly, it was also lower than a study conducted earlier to show prevalence of NTMs among MDR patients, such as; Tabarsi et al. studied 105 suspected MDR-TB subjects in the center of Iran and found $12(11 \%)$ cases of NTM. Aliyu et al. found 69 (15\%) NTM out of 444-mycobacterial positive cultures from consecutive new cases of suspected TB in Nigeria $^{(20)}$. The present study presents similar outcomes on gender as shown in a study done in Korea previously showing higher rates in females than males ${ }^{(21)}$ also showing increased prevalence among older people, while our study, we had mid aged populations $^{(21)}$.

Among the HIV infected, individuals with NTM were lower than the study conducted in USA, ${ }^{(22)}$. While the participants in this study were of middle ages, this was not consistent with other studies showing people of older ages having NTMS, from USA $^{(23)}$. This study showed risk factors associated with NTM infections to be gender and HIV status, while gender proved significant, HIV infection showed no significance risk, in this study' contrary to a study conducted in Spain (2015), which found out that HIV was associated with NTM infections ${ }^{(24)}$.

The study was similar to one conducted in Dhaka, Bangladesh which showed almost similar representation among the gender, while the MTBC infection was observed in all gender in equal numbers, contrary to the global situation as shared that in 2017 close to 6 million adult men contracted TB and around 840,000 died from it. This compares with an estimated 3.2 million adult women who fell ill and almost half a million who died from $\mathrm{TB}^{(25)}$. 
In this study, among these participants with presumed multidrug resistance tuberculosis, the most common NTMs species identified were $M$. Intracellulare, M. Scrofulaceum, among other species. Notably MTBC, was found present at $4.86 \%$, other non-identified mycobacteria species and NTM were also found in distribution.

This study showed Mycobacteria intracellulare (22.1\%) to be prevalent NTM, it was also significant among immunocompromised individuals, especially in HIV people, it is readily available in the soil, water and environment as well as airborne ${ }^{(5)}$ followed by $M$. scrofulaceum among others, contrary to a study of a similar population conducted in Northern India, which found that $\mathrm{M}$. fortuitum was more prevalent followed M. intracellulare ${ }^{(14)}$.

There was co-infection of both TB and NTMs as $14(4.87 \%)$ cases of MTBC were found. Other studies have also showed co-infection rates, a similar in Canada, had NTM/Mtb co-infection of $11 \%,{ }^{(26)}$ and another in USA at $14 \%{ }^{(27)}$. The study showed presence of species of high grade cocci (High GC) at 16\% (microorganisms), these organisms resemble Mycobacteria but are from a different genera (Rhodoccus), these organisms mimic those properties, as acid fastness, thus readily being confused as MTB using the old ZN technology, this shows that if the smear tests were done at the peripheral facilities (16\%) of these would have been captured as TB positive. The high rate of NTM in patients with suspected MDR-TB due to TB treatment failure has important implications for healthcare economics, epidemiology, antimicrobial stewardship, and especially the care and quality of life of the individual patient $^{(15)}$.

\section{Conclusions}

The prevalence of NTMs 287 (12.5\%) which includes $M$. intracellulare $62(21.6 \%)$ and $M$. scrofulaceum 13 (4.5\%) were the most frequently isolated rapid growing mycobacteria among the presumed multidrug resistant tuberculosis participants in Western Kenya. Overall rapid identification and differentiation to species level by molecular assay may help in targeted therapy and management of infections caused by different mycobacterial species and indirectly, it will also help in reducing the developing of antimicrobial drug resistance among NTM isolates in the community.

\section{Limitations}

A limitation to this study, was the inability to test all the possible species of other mycobacteria and other possible NTMs due to the unavailability of a kit, nor using advanced molecular technique.

\section{Declaration}

\section{Ethics approval and consent to participate}

This study was reviewed and approved by the Kenya Medical Research Institute's Scientific and Ethics Review Unit. Protocol Number; KEMRI/SERU/CGHR/084/3410.

\section{Consent for publication}

Not applicable

\section{Availability of data and material}

All data generated or analyzed during this study are included in this published article.

\section{Competing interest}

The authors declare that they have no competing interests.

\section{Funding information}

Funding for this study was provided by the U.S. President's Emergency Plan for AIDS Relief (PEPFAR) through Cooperative Agreement 5U19GH000041 from the U.S. Centers for Disease Control and Prevention (CDC), Division of Global HIV/AIDS and the United States Agency for International Development (USAID). Funding for the sub-study was provided by KEMRI.

\section{Disclaimer}

The findings and conclusions in this reports are those of the authors and do not necessarily represent the official position of the U.S. Centers for Disease Control and Prevention or the Government of Kenya. 


\section{Author's contribution}

Albert Okumu and Steve Wandiga designed the study and drafted the manuscript. Stephen Asito and Isaiah Omondi contributed to the data collection. Christine Ogolla, Clement Shiluli and Wilfred Murithi performed literature search, review and data analysis. Ronald Odero and Timothy Malika carried out sample collection, isolation, culture and identification of isolates. All authors agree to be accountable for all aspects of the work and approved the final version of the manuscript.

\section{Acknowledgments}

We thank the Kenya Ministry of Health (Division of Leprosy, Tuberculosis and Lung Disease and the National AIDS and STI Control Program) and the Kenya Medical Research Institute- Centres for global health research (KEMRI-CGHR), Centers for Disease Control (CDC) in addition to Joseph Orure, Ruth Sitati, Ronald Odero, Christine Agollah and Wilfred Murithi for high quality laboratory testing and data collection.

\section{References}

1. World Health Organization. (2020). Global tuberculosis report 2020. In Global tuberculosis report 2020.

2. Sester, M., C. Giehl, R. McNerney, B. Kampmann, G. Walzl, P. Cuchi, C. Wingfield, C. Lange, G. B. Migliori, A. L. Kritski, A. Meyerhans, A. European Network for global cooperation in the field of and $\mathrm{Tb}$ (2010). "Challenges and perspectives for improved management of HIV/Mycobacterium tuberculosis coinfection." Eur Respir J 36(6): 1242-1247.

3. Johnson, M. M. and J. A. Odell (2014). "Nontuberculous mycobacterial pulmonary infections." J Thorac Dis 6(3): 210-220.

4. Herzmann, C. and C. Lange (2010). "[Infections with non-tuberculous mycobacteria and HIV]." Dtsch Med Wochenschr 135(23): 1192-1197.
5. Agrawal, S., I. Singh, K. J. Kaur, S. R. Bhade, C. L. Kaul and R. Panchagnula (2004). "Comparative bioavailability of rifampicin, isoniazid and pyrazinamide from a four drug fixed dose combination with separate formulations at the same dose levels." Int J Pharm 276(1-2): 41-49.

6. Singh, A. K., A. K. Maurya, J. Umrao, S. Kant, R. A. Kushwaha, V. L. Nag and T. N. Dhole (2013). "Role of GenoType((R)) Mycobacterium Common Mycobacteria/ Additional Species Assay for Rapid Differentiation Between Mycobacterium tuberculosis Complex and Different Species of Non-Tuberculous Mycobacteria." J Lab Physicians 5(2): 8389.

7. Katoch, V. M. (2004). "Infections due to non-tuberculous mycobacteria (NTM)." Indian J Med Res 120(4): 290-304.

8. Ratnatunga, C. N., V. P. Lutzky, A. Kupz, D. L. Doolan, D. W. Reid, M. Field, S. C. Bell, R. M. Thomson and J. J. Miles (2020). "The Rise of Non-Tuberculosis Mycobacterial Lung Disease." Front Immunol 11: 303.

9. Kahkouee, S., E. Esmi, A. Moghadam, M. B. Karam, L. Mosadegh, S. Salek and P. Tabarsi (2013). "Multidrug resistant tuberculosis versus non-tuberculous mycobacterial infections: a CT-scan challenge." Braz J Infect Dis 17(2): 137142.

10. Chopra, K. K., Z. Sidiq, M. Hanif and K. K. Dwivedi (2020). "Advances in the diagnosis of tuberculosis- Journey from smear microscopy to whole genome sequencing." Indian J Tuberc 67(4S): S61S68.

11. Vesga, J. F., T. B. Hallett, M. J. A. Reid, K. S. Sachdeva, R. Rao, S. Khaparde, P. Dave, K. Rade, M. Kamene, E. Omesa, E. Masini, N. Omale, E. Onyango, P. Owiti, M. Karanja, R. Kiplimo, S. Alexandru, V. Vilc, V. Crudu, S. Bivol, C. Celan and N. 
Arinaminpathy (2019). "Assessing tuberculosis control priorities in highburden settings: a modelling approach." Lancet Glob Health 7(5): e585-e595.

12. Masini, E. O., O. Mansour, C. E. Speer, V. Addona, C. L. Hanson, J. K. Sitienei, H. K. Kipruto, M. M. Githiomi and B. N. Mungai (2016). "Using Survival Analysis to Identify Risk Factors for Treatment Interruption among New and Retreatment Tuberculosis Patients in Kenya." PLoS One 11(10): e0164172.

13. Donohue, M. J., J. H. Mistry, J. M. Donohue, K. O'Connell, D. King, J. Byran, T. Covert and S. Pfaller (2015). "Increased Frequency of Nontuberculous Mycobacteria Detection at Potable Water Taps within the United States." Environ Sci Technol 49(10): 6127-6133.

14. Maurya, A. K., V. L. Nag, S. Kant, R. A. Kushwaha, M. Kumar, A. K. Singh and T. N. Dhole (2015). "Prevalence of nontuberculous mycobacteria among extrapulmonary tuberculosis cases in tertiary care centers in Northern India." Biomed Res Int 2015: 465403.

15. Shahraki, A. H., P. Heidarieh, S. Z. Bostanabad, A. D. Khosravi, M. Hashemzadeh, S. Khandan, M. Biranvand, D. E. Schraufnagel and M. Mirsaeidi (2015). "'Multidrug-resistant tuberculosis" may be nontuberculous mycobacteria." Eur J Intern Med 26(4): 279-284.

16. Watterson, S. A., S. M. Wilson, M. D. Yates and F. A. Drobniewski (1998). "Comparison of three molecular assays for rapid detection of rifampin resistance in Mycobacterium tuberculosis." J Clin Microbiol 36(7): 1969-1973.

17. Ahmed, S. T., A. A. Alsudani, R. M. Ali, B. A. Shihab and S. A. Hussein (2016). "Nontuberculosis Mycobacteria: Isolation from clinical samples in Iraq." Int $\mathbf{J}$ Mycobacteriol 5 Suppl 1: S79.
18. Hwang, S. M., M. S. Lim, Y. J. Hong, T. S. Kim, K. U. Park, J. Song, J. H. Lee and E. C. Kim (2013). "Simultaneous detection of Mycobacterium tuberculosis complex and nontuberculous mycobacteria in respiratory specimens." Tuberculosis (Edinb) 93(6): 642-646.

19. Hasegawa, N., T. Miura, K. Ishii, K. Yamaguchi, T. H. Lindner, S. Merritt, J. D. Matthews and S. H. Siddiqi (2002). "New simple and rapid test for culture confirmation of Mycobacterium tuberculosis complex: a multicenter study." J Clin Microbiol 40(3): 908-912.

20. Aliyu, G., S. S. El-Kamary, A. Abimiku, C. Brown, K. Tracy, L. Hungerford and W. Blattner (2013). "Prevalence of nontuberculous mycobacterial infections among tuberculosis suspects in Nigeria." PLoS One 8(5): e63170.

21. Park, S. C., M. J. Kang, C. H. Han, S. M. Lee, C. J. Kim, J. M. Lee and Y. A. Kang (2019). "Prevalence, incidence, and mortality of nontuberculous mycobacterial infection in Korea: a nationwide population-based study." BMC Pulm Med 19(1): 140.

22. Lapinel, N. C., S. E. Jolley, J. Ali and D. A. Welsh (2019). "Prevalence of nontuberculous mycobacteria in HIV-infected patients admitted to hospital with pneumonia." Int J Tuberc Lung Dis 23(4): 491-497.

23. Lin, S., S. Wei, Y. Zhao, J. Lin and Y. Pang (2019). "Epidemiology Of Human Pulmonary Infection With Nontuberculous Mycobacteria In Southeast China: A Prospective Surveillance Study." Infect Drug Resist 12: 3515-3521.

24. Alvaro-Meca, A., L. Rodriguez-Gijon, A. Diaz, A. Gil and S. Resino (2015). "Trends in nontuberculous mycobacterial disease in hospitalized subjects in Spain (1997-2010) according to HIV infection." HIV Med 16(8): 485-493. 


\section{JMSCR Vol||09||Issue||04||Page 186-195||April}

25. WHO (2018). "World Health Organization " Tuberculosis report (http://www.euro.who.int, EUR/TB/FS05).

26. Damaraju, D., F. Jamieson, P. Chedore and T. K. Marras (2013). "Isolation of non-tuberculous mycobacteria among patients with pulmonary tuberculosis in Ontario, Canada." Int J Tuberc Lung Dis 17(5): 676-681.

27. Winthrop, K. L., E. McNelley, B. Kendall, A. Marshall-Olson, C. Morris, M. Cassidy, A. Saulson and K. Hedberg (2010). "Pulmonary nontuberculous mycobacterial disease prevalence and clinical features: an emerging public health disease." Am J Respir Crit Care Med 182(7): 977-982. 